\title{
Reduction of the incidence of development of venous thromboembolism by ultrasound-guided femoral nerve block in total knee arthroplasty
}

\author{
Yusuke Asakura ${ }^{1}$, Hiroki Tsuchiya ${ }^{2}$, Hisatake Mori ${ }^{1}$, Takashi Yano $^{1}$, Yasuhide Kanayama ${ }^{2}$, and \\ Hideki Takagi $^{2}$ \\ Departments of ${ }^{1}$ Anesthesiology, ${ }^{2}$ Orthopedic Surgery, Nagoya Kyoritsu Hospital, Nagoya, Japan
}

Background: Venous thromboembolism (VTE) and the subsequent development of pulmonary embolism (PE) is a major cause of post-operative mortality in total knee arthroplasty (TKA). We evaluated whether the addition of an ultrasound-guided femoral nerve block with general anesthesia affected the incidence in the development of VTE following TKA.

Methods: This was a retrospective non-randomized comparative study with patients assigned to groups based on the surgery date (pre-femoral nerve block versus post-femoral nerve block periods). All anesthesia and medical records of the patients who had undergone computer-navigated TKA in our facility between January 2009 and March 2010 were retrospectively reviewed.

Results: Forty patients were identified; 15 patients underwent computer-navigated TKA under general anesthesia alone (Group G) and 25 patients underwent surgery under general anesthesia combined with ultrasoundguided femoral nerve block (Group F). The incidence of development of VTE post-operatively was significantly lower in Group F ( $\mathrm{P}=0.037)$. Logistic regression analysis identified the use of a femoral nerve block as the most significant variable correlating with the incidence of post-operative development of VTE, and the odds ratio for VTE development in Group G was 3.12 (95\% CI, 0.57-20.56).

Conclusions: We suggest the possibility that the addition of a femoral nerve block on general anesthesia may reduce the incidence of the development of VTE following TKA. (Korean J Anesthesiol 2011; 61: 382-387)

Key Words: Total knee arthroplasty, Ultrasound-guided femoral nerve block, Venous thromboembolism.

Received: February 8, 2011. Revised: April 24, 2011. Accepted: May 17, 2011.

Corresponding author: Yusuke Asakura, M.D., Department of Anesthesiology, Nagoya Kyoritsu Hospital, 1-172, Hokke, Nakagawa-ku, Nagoya 454-0933, Japan. Tel: 81-52-362-5151, Fax: 81-52-353-9105, E-mail: yasakura@kaikou.or.jp

(c) This is an open-access article distributed under the terms of the Creative Commons Attribution Non-Commercial License (http:// creativecommons.org/licenses/by-nc/3.0/), which permits unrestricted non-commercial use, distribution, and reproduction in any medium, provided the original work is properly cited. 


\section{Introduction}

Venous thromboembolism (VTE) and the subsequent development of pulmonary embolism (PE) is a major cause of post-operative mortality in total knee arthroplasty (TKA). Without an appropriate thromboprophylaxis, the prevalence rate can reach as high as $40-80 \%$ for VTE and $2-7 \%$ for PE [1]. Since the introduction of fondaparinux for the prevention of VTE in major orthopedic surgery, the risk has been significantly reduced to less than $15 \%[1,2]$. However, the use of neuroaxial anesthesia techniques in patients who would be expected to receive fondaparinux is controversial [3].

Until the end of June 2009 in our facility, patients had undergone computer-navigated TKA under general anesthesia followed by intravenous fentanyl PCA (patient controlled anesthesia) post-operatively without the use of neuroaxial anesthesia techniques (including epidural and/or spinal anesthesia). For the prophylaxis of post-operative development of VTE, they routinely received fondaparinux following 24 hours after the surgery, However, as of July 2009, we started a combination of single shot ultrasound-guided femoral nerve block on general anesthesia followed by intravenous fentanyl PCA post-operatively. Consequently, of the 40 patients who had undergone computer-navigated TKA between January 2009 and March 2010, 15 patients underwent surgery under general anesthesia alone followed by intravenous fentanyl PCA post-operatively while 25 patients received general anesthesia in combination with ultrasound-guided femoral nerve block followed by intravenous fentanyl PCA post-operatively. Since we switched the anesthesia method to the combination of ultrasound-guided femoral nerve block on general anesthesia for TKA, the incidence of development of VTE has been significantly reduced. We report here our single institutional experience and suggest the possibility that a combination of femoral nerve block on general anesthesia may reduce the incidence of development of VTE following TKA.

\section{Materials and Methods}

We retrospectively reviewed anesthesia charts and medical records of all patients who had undergone computer-navigated TKA in our facility between January 2009 and March 2010. Forty patients were identified, among whom 15 patients underwent general anesthesia alone with sevoflurane, fentanyl and remifentanyl followed by intravenous fentanyl PCA postoperatively (Group G). The other 25 patients underwent computer-navigated TKA under general anesthesia with sevoflurane, fentanyl and remifentanyl in combination with a single shot ultrasound-guided femoral nerve block with a total amount of $25 \mathrm{ml}$ of $0.3-0.5 \%$ ropivacaine followed by intravenous fentanyl PCA (Group F) [4,5]. Additionally, the patients were given rescue doses of ultrasound-guided femoral nerve block with a total amount of $25 \mathrm{ml}$ of $0.3 \%$ ropivacaine at the ward on the first, the second, and the third post-operative day at any time when break through pain to intravenous fentanyl PCA was observed (Group F). Except for the use of ultrasound-guided femoral nerve block, the anesthesia methods were virtually identical between the two groups. In all cases, surgery was carried out with the tourniquet inflated and it was deflated precisely 2 hours after inflation.

All patients started to receive once-daily subcutaneous injection of $1.5 \mathrm{mg}$ of fondaparinux 24 hours after the surgery, together with intermittent pneumatic compression system (IPC) which was started immediately after surgery. As a routine algorithm to rule out the presence of VTE, D-dimer testing in combination with compression ultrasonography was carried out on the third and the seventh post-operative days on the both legs. VTE was considered positive when it was detected ultrasonographically, which was carried out by the skilled cardiovascular ultrasound technicians.

Differences in peri-operative details among the two groups were analyzed using the $\chi^{2}$ test for discrete variables as well as Kruska-Wallis and Wilcoxon rank sum tests for continuous variables. Logistic regression and categorical regression analyses were carried out on the amount of fluid infused, the combination of ultrasound-guided femoral nerve block, concomitant presence of rheumatoid arthritis and the patients' ages on the incidence of post-operative VTE development. These variables were selected and re-examined in a retrograde fashion using logistic regression analysis because statistically significant differences were observed between the two groups only in these four variables. Additionally, the odds ratios of each factor (general anesthesia without the femoral nerve block, the amount of fluids infused, the concomitant presence of rheumatoid arthritis, and the patients' ages) on the incidence of VTE development following TKA including the identification of $95 \%$ confidence intervals (95\% CI) were calculated as previously described elsewhere [6].

\section{Results}

Preoperative patient characteristics are summarized in Table 1. The patients' ages were younger in Group F than those in Group G $(P=0.042)$. Female predominance and body mass index (BMI) were similar in both groups. There were no significant differences in the prevalence of diabetes, hypertension, hyperlipidemia, cardiac disease and renal disease requiring regular hemodialysis between the two groups (Table 1). There was one case of revision TKA in Group G, but the difference was not significant statistically. The prevalence 
Table 1. Characteristics of Patients Preoperatively

\begin{tabular}{|c|c|c|c|}
\hline Measurements & $\begin{array}{l}\text { General anesthesia } \\
\text { alone }(\mathrm{n}=15)\end{array}$ & $\begin{array}{l}\text { General anesthesia and femoral } \\
\text { nerve block }(n=25)\end{array}$ & $\begin{array}{l}\text { Statistical } \\
\text { significance }\end{array}$ \\
\hline Age (yr) & $75.13(66-84)$ & $70.28(55-84)$ & $\mathrm{P}=0.042^{*}$ \\
\hline $\operatorname{Sex}(M / F)$ & $4 / 11$ & $4 / 21$ & $\mathrm{P}=0.41^{\dagger}$ \\
\hline $\operatorname{BMI}\left(\mathrm{kg} / \mathrm{m}^{2}\right)$ & $25.3(19.7-31.56)$ & $24.95(16.2-33.8)$ & $P=0.96^{*}$ \\
\hline ASA (I/II/III) & $1 / 13 / 1$ & $0 / 17 / 8$ & $P=0.06^{\dagger}$ \\
\hline Diabetes (yes/no) & $2 / 13$ & $5 / 25$ & $\mathrm{P}=0.59^{\dagger}$ \\
\hline Hypertension (yes/no) & $10 / 5$ & $12 / 13$ & $\mathrm{P}=0.25^{\dagger}$ \\
\hline Hyperlipidemia (yes/no) & $2 / 13$ & $5 / 20$ & $P=0.59^{\dagger}$ \\
\hline Cardiac disease (yes/no) & $7 / 8$ & $7 / 18$ & $P=0.23^{\dagger}$ \\
\hline Pre-operative antiplatelet and/or anticoagulant therapy (yes/no) & $8 / 7$ & $11 / 14$ & $P=0.57^{\dagger}$ \\
\hline Renal disease requiring hemodialysis (yes/no) & $0 / 15$ & $2 / 23$ & $P=0.16^{\dagger}$ \\
\hline Revision TKA/Primary TKA & $1 / 14$ & $0 / 25$ & $P=0.16^{\dagger}$ \\
\hline Rheumatoid arthritis (yes/no) & $0 / 15$ & $9 / 16$ & $P=0.008^{\dagger}$ \\
\hline Pre-operative glucocorticoid therapy (yes/no) & $0 / 15$ & $4 / 21$ & $P=0.103^{\dagger}$ \\
\hline
\end{tabular}

Data are expressed as means (ranges) or as the number of patients. *Kruskal-Wallis and Wilcoxon rank sum tests. ${ }^{\dagger} \chi^{2}$ test.

Table 2. Peri-operative Variables that May Affect the Incidence in Development of VTE and Post-Operative Outcomes

\begin{tabular}{|c|c|c|c|}
\hline Measurements & $\begin{array}{l}\text { General anesthesia } \\
\text { alone }(n=15)\end{array}$ & $\begin{array}{l}\text { General anesthesia and femoral } \\
\text { nerve block }(n=25)\end{array}$ & $\begin{array}{l}\text { Statistical } \\
\text { significance }\end{array}$ \\
\hline Duration of operation (min) & $188.8(150-272)^{*}$ & $179.5(130-233)^{*}$ & $\mathbf{P}=0.51^{\dagger}$ \\
\hline Intra-operative blood loss (g) & $229.9(45-585)^{*}$ & $171.4(50-385) *$ & $P=0.16^{\dagger}$ \\
\hline Fluid infused (ml) & $2,627(2,100-3,300) *$ & $3,162(1,300-4,000) *$ & $\mathrm{P}=0.0008^{\dagger}$ \\
\hline D-dimer on the third post-operative day $(\mu \mathrm{g} / \mathrm{ml})$ & $5.23(2.3-12.4)^{*}$ & $4.78(2.1-7.8)^{*}$ & $\mathrm{P}=0.92^{\dagger}$ \\
\hline C-reactive protein on the third post-operative day $(\mu \mathrm{g} / \mathrm{ml})$ & $12.76(4.8-22.4) *$ & $15.93(7.2-26.4) *$ & $\mathrm{P}=0.067^{\dagger}$ \\
\hline $\mathrm{D}$-dimer on the seventh post-operative day $(\mu \mathrm{g} / \mathrm{ml})$ & $10.17(3.5-19.4)^{*}$ & $8.83(5.4-16.1)^{*}$ & $\mathrm{P}=0.42^{\dagger}$ \\
\hline C-reactive protein on the seventh post-operative day $(\mu \mathrm{g} / \mathrm{ml})$ & $4.2(0.5-10.8)^{*}$ & $4.44(1.7-10.8) *$ & $P=0.78^{\dagger}$ \\
\hline
\end{tabular}

*Data values are expressed as means (ranges). ${ }^{\dagger}$ Kruskal-Wallis and Wilcoxon rank sum tests.

of rheumatoid arthritis was higher in Group F ( $\mathrm{P}=0.008)$. Accordingly, the number of patients who took glucocorticoids as well as those classified as ASA (American Society of Anesthesiologists) III were higher in Group F, but the difference was not statistically significant.

Peri-operative variables that may affect the incidence of post-operative development of VTE are summarized in Table 2. The duration of the operation as well as the amount of blood loss during the surgery were similar in both groups (Table 2). Presumably owing to the lower blood pressure in Group F, especially at the early period of surgery before the effect of tourniquet pain would have appeared, the amount of fluid infused during the surgery was larger in Group F ( $P$ $=0.0008)$. By contrast, the concentrations of D-dimer on the third post-operative day as well as the seventh post-operative day were similar between the two groups. Reflecting the higher prevalence of rheumatoid arthritis in Group F, C-reactive protein levels on the third and on the seventh post-operative day were slightly higher in Group F, although the differences were not statistically significant.

The development of VTE was routinely evaluated in all patients using compression ultrasonography by skilled cardiovascular ultrasound technicians on the third and seventh post-operative days on both legs, and those diagnosed as having VTE were reported as such immediately. In the compression ultrasonography carried out on the third post-operative day, 7 out of 15 patients were diagnosed as having VTE ultrasonography in Group G, whereas only 4 out of 25 patients were diagnosed in Group F (significantly fewer in Group F, P = 0.037) (Fig. 1). In the cases that developed VTE following TKA, all were detected in the calf region (mostly in the soleal vein) and no proximal type VTE was detected in the observational period. We immediately noticed that the incidence detected on the third post-operative day was quite higher than described previously [2]. However, we also noticed simultaneously that the most studies published thus far detected VTE between the fifth and seventh post-operative days either by the compression ultrasonography or contrast venography $[2,7]$. Interestingly, of the 10 cases that developed VTE on the third post-operative day, 5 out of 10 cases spontaneously resolved on follow-up observations carried out on the seventh post-operative day (Fig. 1) and this was precisely the incidence which we reported 
previously [4]. Since the once-daily subcutaneous injection of fondaparinux had been continued as the initial treatment of VTE, our results support the efficacy of fondaparinux not only in the prevention of, but also in the initial treatment of VTE [8]. Our results also suggest the possibility that the incidence of VTE development following TKA has been underestimated even with the use of fondaparinux, although the dose of fondaparinux we selected is smaller that the standard dose used in the United States and European countries.

To further elucidate what variable(s) most strongly correlated with the incidence of VTE development following TKA postoperatively, we conducted logistic regression analysis on the amount of fluid infused and a categorical regression analysis on the use of a femoral nerve block, the ages of patients and the concomitant presence of rheumatoid arthritis. These analyses identified the combination of femoral nerve block on general anesthesia as the most significant variable that strongly correlated with the prevention of VTE development $(\mathrm{P}=0.0426)$;

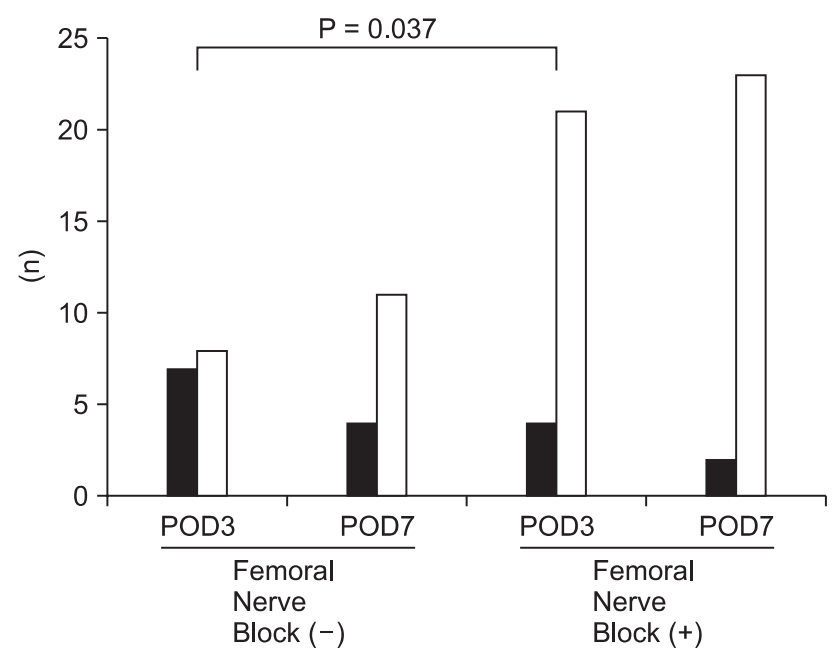

Fig. 1. Number of patients who developed VTE (black bar) and the patients who did not develop VTE (white bar) on the third postoperative day (POD3) and on the seventh post-operative day (POD7) are shown. The incidence in development of VTE is significantly lower in Group F. Note that half the cases that developed VTE on the third post-operative day spontaneously resolved as the administration of fondaparinux was continued. however, the amount of fluid infused $(\mathrm{P}=0.26)$, the patients' ages $(\mathrm{P}=0.297)$ and the concomitant presence of rheumatoid arthritis $(\mathrm{P}=0.908)$ had no effect (Table 3$)$. The calculated odds ratio of general anesthesia alone as compared to the combination of femoral nerve block on the development of VTE was 3.12 (95\% CI, 0.57-20.56), followed by the amount of fluid infused (2.19: 95\% CI, 0.03-79.98), without the concomitant presence of rheumatoid arthritis $(1.72 ; 95 \% \mathrm{CI}, 0.18-38.52)$ and the patients' ages $(0.53 ; 95 \% \mathrm{CI}, 0.02-15.67)$ (Table 3$)$.

\section{Discussion}

Several crucial factors that may affect the incidence of VTE following TKA have been identified thus far, such as the duration of the surgery [9], the use of anticoagulant drugs [10], the use of tourniquet [11] and the use of IPCs [12]. We have shown here that the addition of ultrasound-guided femoral nerve block on general anesthesia may reduce the incidence of development of VTE following TKA.

Similarly, the use of epidural anesthesia during surgery had been previously reported to reduce the incidence of VTE following TKA, presumably owing to the increased blood flow of lower extremities by the effect of sympathectomy [13]. However, this was not the case with the ultrasound-guided femoral nerve block because it is not expected to provide the sufficient level of sympathectomy unless it was performed at the psoas compartment level [14]. Although the precise mechanisms by which the combination of ultrasound-guided femoral nerve block reduced the incidence of VTE following TKA remain to be elucidated, we speculate that with virtually zero pain postoperatively due to excellent analgesic effects of the femoral nerve block on TKA, early mobilization with the assistance of physiotherapists (as early as the first post-operative day) could have prevented the development of VTE in our facility. Although early mobilization with the assistance of a physiotherapist was initiated on the first post-operative day in both groups, it was refused by many patients in Group G because of intense pain during activity, which was not the case for patients in Group F.

The significance of our report is that the addition of a femoral nerve block on general anesthesia had reduced the incidence of

Table 3. Logistic Regression and Categorical Regression Analyses on the Incidence of Development of VTE

\begin{tabular}{|c|c|c|c|}
\hline Measurements & Statistical significance & Odds ratios & $95 \% \mathrm{CI}$ \\
\hline The combination of ultrasound-guided femoral nerve block & $\mathrm{P}=0.0426^{*}$ & $3.12^{\ddagger}$ & $0.57-20.56$ \\
\hline The amount of fluids infused & $P=0.26^{\dagger}$ & 2.19 & $0.03-79.98$ \\
\hline Without the concomitant presence of rheumatoid arthritis & $\mathrm{P}=0.908^{\dagger}$ & $1.72^{\S}$ & $0.18-38.52$ \\
\hline The patients' ages & $\mathrm{P}=0.297^{*}$ & 0.53 & $0.02-15.67$ \\
\hline
\end{tabular}

*The categorical regression analysis. ${ }^{\dagger}$ Logistic regression analysis. ${ }^{\dagger}$ Calculated as, general anesthesia alone/the combination of ultrasoundguided femoral nerve. ${ }^{\S}$ Calculated as, without the concomitant presence of rheumatoid arthritis/the concomitant presence of rheumatoid arthritis. 
development of VTE following TKA even under the routine use of anticoagulants. By contrast, the use of epidural anesthesia for the management of TKA similarly reduced the incidence of post-operative development of VTE. However, the advantage of epidural anesthesia is limited to when anticoagulants can not be used for prophylaxis. This suggests that the impact of the use of anticoagulants as prophylactic therapy for VTE surpasses the benefits of regional anesthesia with respect to the reduction of the incidence of VTE development following TKA [15]. Owing to the relatively small number of patients in our study, there is a possibility of a false positive conclusion. However, the use of femoral nerve block both intra- and postoperatively was particularly efficacious in eliminating pain during activity early in the post-operative period. This conferred superiority to opioid-based general anesthesia alone and/ or in combination with epidural anesthesia, where both were efficacious in eliminating the pain at rest, but not sufficient for pain during activity. Hence, the combination of femoral nerve block might have facilitated early mobilization of the patients with the assistance of a physiotherapist more efficaciously than general anesthesia alone and/or the combination of epidural anesthesia.

During the analysis, we noticed that the incidence in the development of VTE in Group G was quite higher than those reported previously, provided that we had used fondaparinux for the prevention of development of VTE [12]. In addition, the prophylactic effect of fondaparinux in the early post-operative period might have been the same as those in the late postoperative period. However, this fails to account for the reason why subclinical VTE developed in the early post-operative period and spontaneously resolved at the $7^{\text {th }}$ post-operative day. We searched database for kinetic analysis on the incidence and timing of VTE after TKA in recent years, particularly under anticoagulation therapy post-operatively and the studies are very limited. One such large scale retrospective study published in 2009 [16] indentified the second post-operative day as the day where PE (pulmonary embolism) developed most frequently, followed by the first and the third post-operative days. Hence, we speculate that the incidence of development of VTE is usually most frequently seen in the early post-operative period and decreases thereafter, partly owing to the prophylactic effect of fondaparinux in the further development of VTE. In addition, thrombolysis might have taken place in the presence of fondaparinux, which was the primary reason why fondaparinux was recommended as the choice for initial treatment of VTE [8].

Still, the subclinical incidence of VTE in our facility was higher than those reported previously. One possible explanation for the discrepancy we observed was the dose of fondaparinux we have selected. The standard recommended dose of fondaparinux in European countries and in the United
States is once-daily subcutaneous injection of $2.5 \mathrm{mg}$ after major orthopedic surgery [17]. However, we have observed a case with serious gastrointestinal bleeding when $2.5 \mathrm{mg}$ of fondaparinux was used. In this regard, $1.5 \mathrm{mg}$ of fondaparinux can be more safely used in Asian populations without safety issues with the similar efficacy, especially for aged female patients (Asakura Y, Takagi H, unpublished observations).

During the observational period, all VTE cases detected in our facility developed in the calf region and there were no cases that developed PE following VTE. Although the proximal type of VTE is an established cause of pulmonary embolism, there still is controversy regarding the significance of calf vein VTE in the subsequent development of PE. Wang et al. [18] previously reported the precise outcome of VTE cases in the calf region following TKA. They reported 48 cases ( 44 for calf VTE and 4 for popliteal VTE) and only a half (24 cases) were symptomatic. They also reported that no single case developed PE following VTE. However, in some cases, PE may certainly take place even after the development of calf type VTE $[19,20]$. Therefore, it is obvious that even "subclinical" calf type VTE may develop PE without an appropriate thromboprophylaxis.

It was somewhat surprising that the incidence of VTE was significantly lower in Group F, despite the fact that the group consisted of the significantly larger number of patients with rheumatoid arthritis, because it was previously considered to be one of the risk factors for VTE [21]. Our present observations suggest that the multimodal approach to reduce the incidence of VTE which included: complete pain control that enables the early mobilization of the patients with the assistance of physiotherapists, the use of fondaparinux, and the use of IPCs immediately after the surgery, could have reduced the incidence in the development of VTE even in the presence of a single risk factor.

Finally, the limitations of the interpretation of our results are as follows;

1) This was a retrospective comparative study and the background of the patients differs with respect to the following categories; the concomitant presence of rheumatoid arthritis, age and the amount of fluid infused during the surgery. Nonetheless, we were able to identify the use of the femoral nerve as the most significant variable for the prevention of VTE.

2) The number of the patients was relatively small. However, we would like to address the fact that we are unable to conduct a prospective randomized controlled trial owing to the ethical concerns (i.e. fatal outcomes from the development of PE).

In conclusion, we suggest the possibility that addition of a femoral nerve block on general anesthesia reduced the incidence in the development of VTE following TKA. 


\section{References}

1. Bauer KA, Eriksson BI, Lassen MR, Turpie AG. Steering Committee of the Pentasaccharide in Major Knee Surgery Study. Fondaparinux compared with enoxaparin for the prevention of venous thromboembolism after elective major knee surgery. N Engl J Med 2001; 345: 1305-10.

2. Turpie AG, Bauer KA, Eriksson BI, Lassen MR. Fondaparinux vs enoxaparin for the prevention of venous thromboembolism in major orthopedic surgery: a meta-analysis of 4 randomized doubleblind studies. Arch Intern Med 2002; 162: 1833-40.

3. Gogarten W. The influence of new antithrombotic drugs on regional anesthesia. Curr Opin Anaesthesiol 2006; 19: 545-50.

4. Asakura Y, Tsuchiya H, Nieda Y, Yano T, Kato T, Takagi H. Deep vein thrombosis: how long will it remain? J Anesth 2010; 24: 490-1.

5. Asakura Y, Yano T, Takagi H, Tsuchiya H, Kato T. The incidence of development of motor blockade is concentration-dependent, and most studies regarding the continuous FNB after total knee arthroplasty used $0.2 \%$ to $0.3 \%$ ropivacaine. J Arthroplasty $2010 ; 25$ : 836.

6. den Heijer MD, Koster T, Blom HJ, Bos GM, Briet E, Reitsma $\mathrm{PH}$, et al. Hyperhomocysteinemia as a risk factor for deep-vein thrombosis. N Engl J Med 1996; 334: 759-62.

7. Niki Y, Matsumoto H, Hakozaki A, Mochizuki T, Momohara S. Rheumatoid arthritis: a risk factor for deep venous thrombosis after total knee arthroplasty? Comparative study with osteoarthritis. J Orthop Sci 2010; 15: 57-63.

8. Büller HR, Davidson BL, Decousus H, Gallus A, Gent M, Piovella F, et al. Fondaparinux or enoxaparin for the initial treatment of symptomatic deep venous thrombosis. Ann Intern Med 2004; 140: 867-73.

9. Sharrock NE, Ranawat CS, Urquhart B, Peterson M. Factors influencing deep vein thrombosis after total hip arthroplasty under epidural anesthesia. Anesth Analg 1993; 76: 765-71.

10. Sharrock NE, Brien WW, Salvati EA, Mineo R, Garvin K, Sculco TP. The effect of intravenous fixed-dose heparin during total hip arthroplasty on the incidence of deep-vein thrombosis: a randomized, double-blinded trial in patients operated on with epidural anesthesia and controlled hypotension. J Bone Joint Surg
Am 1990; 72: 1456-61.

11. Abdel-Salam A, Eyres KS. Effects of tourniquet during total knee arthroplasty: A prospective randomized study. J Bone Joint Surg Br 1995; 77: 250-3.

12. Woolson ST. Intermittent pneumatic compression prophylaxis for proximal deep venous thrombosis after total hip replacement. J Bone Joint Surg Am 1996; 78: 1735-40.

13. Sharrock NE, Haas SB, Hargett MJ, Urquhart B, Insall JN, Scuderi G. Effects of epidural anesthesia on the incidence of deep-vein thrombosis after total knee arthroplasty. J Bone Joint Surg Am 1991; 73: 502-6.

14. Asakura Y, Kandatsu N, Kato N, Sato Y, Fujiwara Y, Komatsu T. Ultra-sound guided sciatic nerve block combined with lumbar plexus block for infra-inguinal artery bypass graft surgery. Acta Anaesthesiol Scand 2008; 52: 721-2.

15. Hu S, Zhang ZY, Hua YQ, Li J, Cai ZD. A comparison of regional and general anaesthesia for total replacement of the hip or knee; A meta-analysis. J Bone Joint Surg Br 2009; 91: 935-42.

16. Clayton RA, Gaston P, Watts AC, Howie CR. Thromboembolic disease after total knee replacement: Experiences of 5100 cases. Knee 2009; 16: 18-21.

17. Singelyn FJ, Verheyen C, Piovella F, Van Aken HK, Rosencher N; EXPERT Study Investigators. The safety and efficacy of extended thromboprophylaxis with fondaparinux after major orthopedic surgery of the lower limb with or without a neuroaxial or deep peripheral nerve catheter: The EXPERT Study. Anesth Analg 2007; 105: 1540-7.

18. Wang CJ, Wang JW, Weng LH, Hsu CC, Lo CF. Outcome of calf deepvein thrombosis after total knee arthroplasty. J Bone Joint Surg Br 2003; 85: 841-4.

19. Pellegrini VD Jr, Langhans MJ, Totterman S, Marder VJ, Francis CW. Embolic complications of calf thrombosis following total hip arthroplasty. J Arthroplasty 1993; 8: 449-57.

20. Gottlieb RH, Widjaja J, Mehra S, Robinette WB. Clinically important pulmonary emboli: Does calf vein US alter outcomes? Radiology 1999; 211: 25-9.

21. Matta F, Singala R, Yaekoub AY, Najjar R, Stein PD. Risk of venous thromboembolism with rheumatoid arthritis. Thromb Haemost 2009; 101: 134-8. 